\title{
Cortical muscle control of spontaneous movements in human neonates(Dissertation_全文)
}

AUTHOR(S):

Kanazawa, Hoshinori

\section{CITATION:}

Kanazawa, Hoshinori. Cortical muscle control of spontaneous movements in human neonates. 京都大学, 2014, 博士(医学)

ISSUE DATE:

2014-07-23

URL:

https://doi.org/10.14989/doctor.k18506

\section{RIGHT:}

登録版にはWiley Onlinw Libraryの最終版へのリンクが記載されなけれ ばならない。また、最終版への改訂や変更は許可されていない。最 終版へのリンク

http://onlinelibrary.wiley.com/doi/10.1111/ejn.12612/abstract; 許諾条 件により全文は2015-05-15に公開 


\title{
Cortical muscle control of spontaneous movements in human neonates
}

\author{
（新生坚運動時における \\ 大脳皮質由来の筋活動について）
}

金沢 星慶 
European Journal of Neuroscience (published online: 15 May 2014)

Title: Cortical muscle control of spontaneous movements in human neonates

Hoshinori Kanazawa ${ }^{1,4}$, Masahiko Kawai ${ }^{1}$, Takahiro Kinai ${ }^{2}$, Kougorou Iwanaga ${ }^{1}$, Tatsuya Mima $^{3}$, and Toshio Heike ${ }^{1}$

${ }^{1}$ Department of Pediatrics, Graduate School of Medicine, Kyoto University, 606-8507 Kyoto, Japan

${ }^{2}$ Morinomiya University of Medical Sciences, 559-8611 Osaka, Japan

${ }^{3}$ Human Brain Research Center, Kyoto University, 606-8507 Kyoto, Japan

${ }^{4}$ Research Fellow, the Japan Society for the Promotion of Science, 102-0083 Tokyo, Japan

Runnning title: Neonatal Cortical Muscle Control

Key word: coherence, EEG, EMG, Granger causality, Neonate

Correspondence to: Hoshinori Kanazawa

Department of Pediatrics, Graduate School of Medicine, Kyoto University

54 Kawara-machi, Shogoin, Sakyo-ku, Kyoto 606-8507, Japan

Telephone: 81-75-751-3290, Fax: 81-75-752-2361, E-mail: Hoshi@ kuhp.kyoto-u.ac.jp 


\begin{abstract}
Anatomical studies show the existence of corticomotor neuronal projections to the spinal cord before birth, but whether the primary motor cortex (M1) drives muscle activity in neonate "spontaneous" movements is unclear. To investigate this issue, we calculated corticomuscular coherence (CMC) and Granger causality in neonates. CMC is widely used as an index of functional connectivity between M1 and limb muscles, and Granger causality is used across many fields of science to detect the direction of coherence. To calculate CMC and Granger causality, we used electroencephalography (EEG) to measure activity over the cortical region that governs leg muscles, and surface electromyography (EMG) over the right and left tibialis anterior muscles, in 15 healthy term and preterm neonates, during spontaneous movements without any external stimulation. We found that 17 leg muscles (10 right, 7 left) in 12 neonates showed significant $\mathrm{CMC}$, whose magnitude significantly correlated with postnatal age only in the beta frequency band. Further analysis revealed Granger causal drive from EEG to EMG in 14 leg muscles. Our findings suggest that M1 drives muscle activity when neonates move their limbs. Moreover, the positive correlation between $\mathrm{CMC}$ magnitude and postnatal age suggests that corticomuscular communication begins to develop during the neonatal stage. This process may facilitate sensory-motor integration and activity-dependent development.
\end{abstract}


Introduction

Human neonates move their bodies freely even soon after birth (van der Meer et al., 1995; von Hofsten, 2004). Some of these movements appear to be purposeful, spontaneous actions, while others resemble automatic responses and primitive reflexes. The question of whether a neonate's movement is under spontaneous control remains unanswered.

Cortical control of muscle activity is a physiologic prerequisite for the generation of spontaneous action. The development of the corticospinal tract (CST) has thus been studied extensively. Animal experiments and autopsies in humans show that the CST exists anatomically at birth, and that its microstructure changes dramatically as it matures (Kuypers, 1962; Eyre et al., 2000; ten Donkelaar et al., 2004). Furthermore, transcranial magnetic stimulation (TMS) of neonatal motor cortex can evoke motor unit discharges via the CST, with conduction velocities that increase during infancy and reflect neuronal myelinization (Armand et al., 1994; Müller et al., 1994; Olivier et al., 1997; Eyre et al., 2000; Fietzek et al., 2000; Eyre et al., 2001). Animal studies have provided critical evidence that this developmental process requires the activity of both motor cortex and muscles, referred to as activity-dependent development (Martin et al., 2004; Martin, 2005; Martin et al., 2007; Chakrabarty et al., 2009; Friel et al., 2012). Although previous studies regarding human neonatal movements have suggested a temporal association between electroencephalography (EEG) signals and limb movements (Milh et al., 2007; Thorsteinsson et al., 2010), the functional communication between spontaneous cortical and muscle activity, and its direction, has not been studied. 
Corticomuscular coherence (CMC) quantitatively measures the degree of cortical control over muscle activity using brain-activity data like EEG and electromyography (EMG). These methods are non-invasive and suitable for investigating natural spontaneous movements (Conway et al., 1995; Halliday et al., 1995; Halliday et al., 1998; Mima \& Hallett, 1999a). Because synchronization of neural oscillatory activity is thought to reflect functional communication (Gray et al., 1989), CMC (mainly in the beta frequency band, 14-32 Hz) likely reflects functional connectivity within the motor system, in particular efferent commands from the cortex to muscle. Although it is assumed that neural oscillatory activity in the corticospinal system has not matured in young children (Farmer et al., 2007; James et al., 2008; Petersen et al., 2010), Graziadio et al. (2010) reported significant CMC in this age group (4-12 years of age) and its increase in amplitude with age. Thus, even the human neonate may have significant CMC.

CMC is measured as a correlation between signals, but it does not provide directional information. Several computational methods have been proposed to solve this problem, and Granger causality, based on predictability, is one of the most powerful and widely used methods (Granger, 1969; Seth, 2008, 2010).

To test the direction of functional corticomuscular communication during spontaneous movements in the early developmental stage, we calculated the CMC and Granger causality of EEG and EMG signals from healthy term and preterm neonates at term-equivalent ages.

Materials and Methods

Subjects 
Fifteen healthy term and preterm neonates (eight male, seven female; full term: 5, preterm: 10), aged 36-42 weeks (post-conception), participated in this experiment 5-67 days following their birth at Kyoto University Hospital. None of the subjects had neurological impairments, including intraventricular hemorrhage, chromosomal abnormalities, or congenital infection. This study was conducted in accordance with the Declaration of Helsinki. Ethics approval was obtained from Kyoto University Graduate School and Faculty of Medicine Ethics Committee, and written informed parental consent was obtained before each procedure.

\section{Recording and preprocessing}

EEG was recorded from the primary sensorimotor area for leg muscles $(\mathrm{Cz}$ according to the 10/20 international system) with four surrounding electrodes (inter-electrode distance: $2 \mathrm{~cm}$ to $\mathrm{Cz}$ ) (Mima \& Hallett, 1999b). The reference electrode for the recording was placed at FPz, and the ground electrode was placed at the nasion. To increase the spatial resolution of the EEG signal, the Hjorth derivation was applied, where the mean voltage of four surrounding electrodes were used as a reference (Figure 1).

EMG was recorded from the right and left tibialis anterior (TA) using pairs of electrodes. Ag/AgCl active electrodes (EasyCap, Herrsching, Germany) were used for both EEG and EMG. These signals were amplified, analog-filtered (band-pass: 0.5-300 $\mathrm{Hz}$ ), digitized at a sampling rate of $1000 \mathrm{~Hz}$, and stored on an EEG 2100 (Nihon Kohden, Tokyo, Japan). The EEG and EMG signals were then filtered using a band-pass of 5-55 $\mathrm{Hz}$ and 5-300 Hz, respectively. A low-pass filter at $55 \mathrm{~Hz}$ was applied after EMG rectification. 
Neonate recordings were made under calm and awake conditions in the experimenter's arm (approximately $60 \mathrm{~min}$ in total, Figure 2). Awareness was confirmed by checking whether the EEG showed a trace alternant pattern, which indicates the infantile sleep state (Eyre, 1992). Spontaneous muscle contractions longer than 2 s were selected separately for the right and left TA. The period of muscle contraction was defined as the time in which the EMG amplitude was two standard deviations (SD) greater than that measured during the baseline resting period. Artifact-free stable periods (duration: $1 \mathrm{~s}$ ) were extracted, excluding the initial and final 0.5-s transition periods (Figure 3). Finally, $300 \mathrm{~s}$ of stable contraction periods were selected for the left and right TA in each neonate as the EEG and EMG signals for analysis. EEG and rectified EMG signals were segmented into epochs of $0.5 \mathrm{~s}$, detrended, and windowed with a Hanning window.

\section{Analysis}

Statistical analyses were performed using Matlab software (Matlab version 8.1, Mathworks, Natick, MA, USA).

First, to quantitatively measure corticomuscular functional connectivity, the CMC was computed. The CMC is an extension of Pearson's correlation coefficient in the frequency domain, and ranges from 0 to 1 , with 1 indicating identical signals (i.e., a perfect correlation). The CMC between signal $\mathrm{i}$ and signal $\mathrm{j}$ can be obtained by normalizing the square of the cross-spectra by the auto-spectra, using the following equation:

$$
\operatorname{Coh}_{i j}(f)=\operatorname{Coh}_{j i}(f)=\frac{\left|S_{i j}(f)\right|^{2}}{S_{i i}(f) S_{j j}(f)}
$$


In this equation, $S_{i i}(f), S_{j j}(f)$ and $S_{i j}(f)$ are values of auto- and cross-spectra at a frequency $f$, which are calculated using a fast Fourier transformation.

Second, to test the relationship between development and corticomuscular functional connectivity, the Pearson's correlation coefficient between the CMC and postnatal age was determined, after applying an arc hyperbolic tangent transformation on the CMC. This relationship was determined using a weighted correlation coefficient, with the numbers of legs on each subject as the weight.

Third, to investigate the direction of significant corticomuscular connectivity, we calculated the spectral Granger causality using a bivariate auto-regression model of order 50 (Seth, 2010). The bivariate auto-regression model can be described as

$$
X_{t}=\sum_{k=1}^{p} A_{k} X_{t-k}+E_{t}
$$

where $X_{t}$ is the two-dimensional vector representing signal $i$ and signal $j$ at the time $t, A_{k}$ is the model coefficient, $p$ is the model order, and $E_{t}$ is white noise. Spectral properties of the signal $X(f)$ can be obtained by z-transformation of the above equation

$$
X(f)=A(f)^{-1} E(f)=H(f) E(f)
$$

where $H(f)$ is a transfer function of the system. The spectral matrix $S(f)$ is then calculated using the formula with the residual white noise $\Sigma$ :

$S(f)=X(f) X(f)^{*}=H(f) \Sigma H(f)^{*}$

Finally, spectral Granger causality, which is calculated for all frequencies from signal $\mathrm{j}$ to signal $\mathrm{i}$ is defined as

$$
I_{j \rightarrow i}(f)=-\ln \left(1-\frac{\left(\Sigma_{j j}-\frac{\Sigma_{i j}^{2}}{\Sigma_{i i}}\right)\left|H_{i j}(f)\right|^{2}}{S_{i i}(f)}\right)
$$


A significant CMC and Granger causality was defined as being greater than the 95\% confidence limit, which was computed using a repeated permutation test (100 runs), randomly shuffling EEG and EMG data (Brovelli et al., 2004; Maris et al., 2007; Bollimunta et al., 2011). The 95\% confidence limit for CMC in this study was 0.005 , and Granger causality was calculated individually for each subject.

Results

All 15 neonates completed the study, and $300 \mathrm{~s}$ of data representing muscle contractions were selected for each TA of each neonate. Since a preliminary analysis revealed neither term vs. preterm nor right vs. left differences in $\mathrm{CMC}$, and number of subjects was too small to perform an analysis of subgroups, data from all subjects was pooled for the analysis. The power spectra of EEG and EMG peaked at $9 \mathrm{~Hz}$ (range: 8 to $11 \mathrm{~Hz}$ ) and 9 $\mathrm{Hz}$ (range: 5 to $22 \mathrm{~Hz}$ ) respectively, and both of them decreased gradually to $50 \mathrm{~Hz}$ (Figure 4A, B). Twelve out of the 15 neonates (10 right and seven left TAs) showed significant CMCs greater than 0.005 (mean: 0.0077, range: 0.006-0.008) (Figure 4C). The frequencies that contained the peak CMC values were in the alpha band ( 8 to $12 \mathrm{~Hz}$ in six TAs), beta band (13 to $30 \mathrm{~Hz}$ in seven TAs), and gamma band (30 to $55 \mathrm{~Hz}$ in four TAs). Multiple peaks in CMC were observed in five TAs. However, the secondary peaks were very small compared to the primary peaks, and thus for the analysis, we focused on the maximal peaks for each TA. Postnatal age and CMC magnitude were significantly and positively correlated $(\mathrm{r}=0.67, \mathrm{p}=0.0055$, Figure 5$)$. We calculated the correlation between CMC amplitude and age for each frequency separately. There was a significant correlation in the beta band $(\mathrm{r}=0.96, \mathrm{p}=0.0028)$, but not in the alpha or gamma bands ( $\mathrm{r}$ 
$=0.35, \mathrm{p}=0.50 ; \mathrm{r}=0.63, \mathrm{p}=0.37$, respectively). In contrast, there was no significant correlation between postnatal age and power of EEG or EMG $(r=-0.03, p=0.90 ; r=$ $0.22, \mathrm{p}=0.40$, respectively).

We then tested the differences in peak frequencies (median: range) between CMC (18 Hz: 8 to $51 \mathrm{~Hz})$, EEG (9 Hz: 8 to $11 \mathrm{~Hz})$, and EMG (9 Hz: 5 to $22 \mathrm{~Hz})$. Bartlett's test confirmed that these data were not normally distributed, therefore we used the non-parametric Friedman test, and a post hoc Wilcoxon rank sum test. We found significant differences for CMC vs. EEG ( $p=0.002)$, CMC vs. EMG, $(p=0.007)$, but not for EEG vs. EMG, $(\mathrm{p}=0.48)$.

We performed a spectral Granger causality analysis for the 17 TAs that showed significant CMC. Fourteen out of them showed a descending Granger causal drive from the cortex to the TA (seven right and seven left TAs). Six of these TAs (four right and two left TAs) also showed ascending Granger causality from EMG to EEG (i.e., bidirectional connectivity). The remaining three right TAs showed only ascending Granger causality from EMG to EEG (Figure 6).

\section{Discussion}

Behavioral research has proposed that human neonates move purposefully (van der Meer et al., 1995; von Hofsten, 2004). In this study, we used electrophysiological techniques to uncover two important physiological findings regarding neonatal spontaneous movements. First, by calculating CMC, we demonstrated functional connectivity between the cortex and muscle. Second, by calculating the Granger causality for significant CMCs, we showed that corticomuscular connectivity predominantly descends from the cortex to 
muscles. These results indicate that neonatal cortex drives muscle activity, which in turn suggests that neonates move their limbs spontaneously besides reflex-based movementes.

Because the descending CST is a common pathway for spontaneous movement (Haggard, 2008), spontaneous and unspontaneous movement can be detected when muscle activity is driven from the motor cortex via the CST. The CST already exists during early postnatal stages, and even develops prenatally in humans (Koh \& Eyre, 1988; Eyre et al., 2000; ten Donkelaar et al., 2004). The microstructure of the CST changes considerably during postnatal development, with increases to the number of synapses and dendrites, and localization of cortical neuron terminations. Animal studies show that these changes require spontaneous cortical activity, described as activity-dependent development (Martin et al., 2004; Martin, 2005; Martin et al., 2007; Chakrabarty et al., 2009; Friel et al., 2012). In fact, there has been some controversy surrounding the maturation pattern of the CST. Some animal studies have suggested that maturation of cortical functional connectivity to limb muscles is not established until 2.5 months (Armand et al., 1997; Olivier et al., 1997). However, the maturation pattern of limb corticospinal tracts might differ across species. This is based on reports that TMS or electrical stimulation to the motor cortex can evoke upper and lower muscle discharge, through CST, in human neonates (Koh \& Eyre, 1988; Khater-Boidin \& Duron, 1991; Santiago-Rodríguez et al., 2009). Consequently, it is conceivable that functional connectivity between motor cortex and muscles exists even in the human neonatal stage. Our findings provide supporting evidence for the neonatal cortical control of muscle activity, using CMC and Granger causality. 
$\mathrm{CMC}$ has been shown to reflect corticomuscular functional connectivity in a number of studies; however, the study of neonatal CMC has been limited because of technical difficulties. For example, James et al. (2008) tried to measure neonatal CMC using the grasp reflex in a population study among infants, children, and adults and believed that it might have actually reflected a spinal reflex. To solve this problem, we observed neonatal movement without any stimulation, thus the CMC in our study reflected spontaneous movements, which would have been impossible to induce using external cortical stimulation with TMS.

It has been reported that $\mathrm{CMC}$ increases in amplitude with age (Graziadio et al., 2010; Kamp et al., 2013). Consistent with this, James et al. (2008) showed that young children below 10 years of age had a featureless peak frequency of CMC. In our study, the neonatal $\mathrm{CMC}$ also showed low amplitude and a variation in peak frequencies when compared with young children or adult data from previous studies (Graziadio et al., 2010; Ushiyama et al., 2011; Kamp et al., 2013). These features imply that oscillatory corticomuscular functional connectivity is immature during developmental stage, which is consistent with the findings of EMG-EMG coherence studies (Farmer et al., 2007; Petersen et al., 2010). Further support for this notion is the positive correlation between CMC and postnatal age, suggesting that activity-dependent development of the corticospinal system occurs during infancy.

Another possible reason for the variation in peak CMC frequencies might be the type of muscle contraction type. A number of previous studies have focused on CMC in the beta range during isometric muscle contraction, but CMC in the gamma band has also been observed in certain situations. Strong muscle contraction shifts CMC into the 
gamma range (Brown et al., 1998; Mima et al., 1999), and the same phenomenon is also observed during dynamic contraction (Omlor et al., 2007). On the other hand, the role of CMC in the alpha band has not been established (Mima \& Hallett, 1999a). Taken together, the findings of these previous studies suggest that the frequency of $\mathrm{CMC}$ reflects muscle dynamics. In this study, CMC may have included some different types of muscle contraction; however, it is impossible to obtain homogeneous movement in a neonatal population. We also observed that $\mathrm{CMC}$ in the beta band correlated with age more strongly than $\mathrm{CMC}$ at any other frequency band. A cluster of alpha frequency was observed at less than 40 days after birth, gamma frequency clustered at greater than 40 days after birth, and CMC at the beta frequency varied across age. These results support the idea that $\mathrm{CMC}$ in the beta band has a functional role when compared with other frequency bands, even in developmental processes.

Next, we investigated the direction of corticomuscular connectivity with Granger causality, a method proven useful in numerous research fields, including neuroscience (Granger, 1969; Kamiński et al., 2001; Seth, 2008, 2010). Granger causality analysis revealed that connectivity was driven in a descending manner (i.e., from EEG to EMG) in 14 out of 17 TAs, suggesting that the cortex drives muscle activity even in neonates. Until now, researchers have assumed that neonatal movements are spontaneous, while offering only indirect evidence regarding the physiological mechanisms to support this assumption. In contrast, our study found significant CMC in a descending direction, which provides strong physiological evidence that spontaneous movements in neonates are under cortical control. These descending muscle controls might play an important role in activity-dependent development in the CST. 
In addition to a descending drive, we found bidirectional connectivity in six TAs. This is not unusual, and Witham et al. (2011) observed a similar bidirectionality in adult CMC. These findings imply the existence of both cortical control of muscle activity and sensory feedback corresponding to muscle contraction, consistent with animal experiments (Baker et al., 2006). Finding bidirectional connectivity in only six TAs may also imply that neonatal corticomuscular functional connectivity is immature. In a small number of cases, we found only unidirectional ascending Granger causality, which we interpret as sensory feedback from the TA. If we assume that unidirectional ascending Granger causality implies a lack of cortical motor command, it follows that neonatal muscle activity with unidirectional ascending Granger causality might be considered a spinal reflex. Another possibility is that the amount of descending drive in these TAs was too small to detect because of immaturity of the CST. Future research is needed to confirm whether neonatal reflex activity exhibits only ascending Granger causality. As an addendum, it should be noted that Granger causality is defined here in the statistical sense, which means that "Granger causal drive" does not equate to a demonstration of causality per se.

In clinical neonatal care, magnetic resonance imaging or other clinical anatomical techniques are used to detect major neural impairments. However, preterm infants without major neuronal impairment are well known to be at high risk for developmental motor difficulties. For this reason, micro-anatomical or functional impairments may exist that conventional methods cannot detect. In a recent TMS study, Pitcher et al. (2012a, 2012b) reported that children born preterm showed reduced corticomotor plasticity and excitability. Logically, the assessment of corticomuscular 
functional connectivity is clinically important for the care of these at-risk neonates and their future motor development. However, few studies have used TMS in human neonates, presumably because of ethical considerations. Clinically, neonatal brain function or development is assessed predominantly using EEG. EEG spectral power, which reflects rhythmic activity of corticomotor neurons, is known to change gradually after birth (Khazipov \& Luhmann, 2006; Biagioni et al., 2007; Tolonen et al., 2007). Moreover, EMG spectral power that reflects the activity of motor units also changes during human development (Hadders-Algra et al., 1992; Gibbs et al., 1997; Farmer et al., 2007; Petersen et al., 2010). Both EEG and EMG might interact with each other while changing their oscillations during infancy. CMC and Granger causality might help determine how these neural groups communicate with each other through synchronous oscillation. Assuming that bidirectional connectivity reflects cortical muscle control and sensory feedback, this would indicate the appearance of sensory-motor integration, which is a profoundly important stage of sensory-motor development in infants. The importance of the non-invasive nature of the tests used here cannot be understated. These results regarding neonatal neural communication, in conjunction with the non-invasive methods, provide firm evidence that this technique is clinically viable for the assessment of neurological motor dysfunction in human neonates.

\section{Conclusion}

This study provided evidence for descending cortical control of motor activity in human neonates, using CMC and Granger causality between EEG and EMG. Critically, CMC significantly correlated with postnatal age only in the beta frequency band, implying a 
relationship between $\mathrm{CMC}$ and neural maturation during neonatal development. These results also suggest that corticomuscular communication begins to develop during the neonatal stage. This may facilitate sensory-motor integration and activity-dependent development.

\section{Acknowledgement}

H.K. was supported by the Japan Society for the Promotion of Science (Grant-in-Aid for JSPS fellows, 25-2682). We thank all the infants and their parents who participated in this study. We also thank the staff at Kyoto University Hospital.

\section{References}

Armand, J., Edgley, S.A., Lemon, R.N. \& Olivier, E. (1994) Protracted postnatal development of corticospinal projections from the primary motor cortex to hand motoneurones in the macaque monkey. Exp Brain Res, 101, 178-182.

Armand, J., Olivier, E., Edgley, S.A. \& Lemon, R.N. (1997) Postnatal development of corticospinal projections from motor cortex to the cervical enlargement in the macaque monkey. J Neurosci, 17, 251-266.

Baker, S.N., Chiu, M. \& Fetz, E.E. (2006) Afferent encoding of central oscillations in the monkey arm. J Neurophysiol, 95, 3904-3910.

Biagioni, E., Frisone, M.F., Laroche, S., Kapetanakis, B.A., Ricci, D., Adeyi-Obe, M., Lewis, H., Kennea, N., Cioni, G., Cowan, F., Rutherford, M., Azzopardi, D. \& Mercuri, E. (2007) Maturation of cerebral electrical activity and development of cortical folding in young very preterm infants. Clin Neurophysiol, 118, 53-59.

Bollimunta, A., Mo, J., Schroeder, C.E. \& Ding, M. (2011) Neuronal mechanisms and attentional modulation of corticothalamic $\alpha$ oscillations. J Neurosci, 31, 4935-4943.

Brovelli, A., Ding, M., Ledberg, A., Chen, Y., Nakamura, R. \& Bressler, S.L. (2004) Beta oscillations in a large-scale sensorimotor cortical network: directional influences revealed by Granger causality. Proc Natl Acad Sci U S A, 101, 9849-9854.

Brown, P., Salenius, S., Rothwell, J.C. \& Hari, R. (1998) Cortical correlate of the Piper rhythm in humans. J Neurophysiol, 80, 2911-2917. 
Chakrabarty, S., Friel, K.M. \& Martin, J.H. (2009) Activity-dependent plasticity improves M1 motor representation and corticospinal tract connectivity. J Neurophysiol, 101, 1283-1293.

Conway, B.A., Halliday, D.M., Farmer, S.F., Shahani, U., Maas, P., Weir, A.I. \& Rosenberg, J.R. (1995) Synchronization between motor cortex and spinal motoneuronal pool during the performance of a maintained motor task in man. The Journal of physiology, 489 ( Pt 3, 917-924.

Eyre, J.A. (1992) The Neurophysiological examination of the newborn infant. Mac Keith.

Eyre, J.A., Miller, S., Clowry, G.J., Conway, E.A. \& Watts, C. (2000) Functional corticospinal projections are established prenatally in the human foetus permitting involvement in the development of spinal motor centres. Brain, 123 ( Pt 1), 51-64.

Eyre, J.A., Taylor, J.P., Villagra, F., Smith, M. \& Miller, S. (2001) Evidence of activity-dependent withdrawal of corticospinal projections during human development. Neurology, 57, 1543-1554.

Farmer, S.F., Gibbs, J., Halliday, D.M., Harrison, L.M., James, L.M., Mayston, M.J. \& Stephens, J.A. (2007) Changes in EMG coherence between long and short thumb abductor muscles during human development. J Physiol, 579, 389-402.

Fietzek, U.M., Heinen, F., Berweck, S., Maute, S., Hufschmidt, A., Schulte-Mönting, J., Lücking, C.H. \& Korinthenberg, R. (2000) Development of the corticospinal system and hand motor function: central conduction times and motor performance tests. Dev Med Child Neurol, 42, 220-227.

Friel, K., Chakrabarty, S., Kuo, H.C. \& Martin, J. (2012) Using motor behavior during an early critical period to restore skilled limb movement after damage to the corticospinal system during development. J Neurosci, 32, 9265-9276.

Gibbs, J., Harrison, L.M. \& Stephens, J.A. (1997) Cross-correlation analysis of motor unit activity recorded from two separate thumb muscles during development in man. J Physiol, 499 ( Pt 1), 255-266.

Granger, C.W. (1969) Investigating causal relations by econometric models and cross-spectral methods. Econometrica: Journal of the Econometric Society, 424-438.

Gray, C.M., König, P., Engel, A.K. \& Singer, W. (1989) Oscillatory responses in cat visual cortex exhibit inter-columnar synchronization which reflects global stimulus properties. Nature, 338, 334-337.

Graziadio, S., Basu, A., Tomasevic, L., Zappasodi, F., Tecchio, F. \& Eyre, J.A. (2010) Developmental tuning and decay in senescence of oscillations linking the corticospinal system. J Neurosci, 30, 3663-3674.

Hadders-Algra, M., Van Eykern, L.A., Klip-Van den Nieuwendijk, A.W. \& Prechtl, H.F. (1992) Developmental course of general movements in early infancy. II. EMG correlates. Early Hum Dev, 28, 231-251. 
Haggard, P. (2008) Human volition: towards a neuroscience of will. Nat Rev Neurosci, 9, 934-946.

Halliday, D.M., Conway, B.a., Farmer, S.F. \& Rosenberg, J.R. (1998) Using electroencephalography to study functional coupling between cortical activity and electromyograms during voluntary contractions in humans. Neuroscience letters, 241, 5-8.

Halliday, D.M., Rosenberg, J.R., Amjad, A.M., Breeze, P., Conway, B.A. \& Farmer, S.F. (1995) A framework for the analysis of mixed time series/point process data--theory and application to the study of physiological tremor, single motor unit discharges and electromyograms. Prog Biophys Mol Biol, 64, 237-278.

James, L.M., Halliday, D.M., Stephens, J.A. \& Farmer, S.F. (2008) On the development of human corticospinal oscillations: age-related changes in EEG-EMG coherence and cumulant. Eur J Neurosci, 27, 3369-3379.

Kamiński, M., Ding, M., Truccolo, W.A. \& Bressler, S.L. (2001) Evaluating causal relations in neural systems: granger causality, directed transfer function and statistical assessment of significance. Biol Cybern, 85, 145-157.

Kamp, D., Krause, V., Butz, M., Schnitzler, A. \& Pollok, B. (2013) Changes of cortico-muscular coherence: an early marker of healthy aging? Age (Dordr), 35, 49-58.

Khater-Boidin, J. \& Duron, B. (1991) Postnatal development of descending motor pathways studied in man by percutaneous stimulation of the motor cortex and the spinal cord. Int $\mathbf{J}$ Dev Neurosci, 9, 15-26.

Khazipov, R. \& Luhmann, H.J. (2006) Early patterns of electrical activity in the developing cerebral cortex of humans and rodents. Trends Neurosci, 29, 414-418.

Koh, T.H. \& Eyre, J.A. (1988) Maturation of corticospinal tracts assessed by electromagnetic stimulation of the motor cortex. Arch Dis Child, 63, 1347-1352.

Kuypers, H.G. (1962) Corticospinal connections: postnatal development in the rhesus monkey. Science, 138, 678-680.

Maris, E., Schoffelen, J.M. \& Fries, P. (2007) Nonparametric statistical testing of coherence differences. J Neurosci Methods, 163, 161-175.

Martin, J.H. (2005) The corticospinal system: from development to motor control. Neuroscientist, $11,161-173$.

Martin, J.H., Choy, M., Pullman, S. \& Meng, Z. (2004) Corticospinal system development depends on motor experience. J Neurosci, 24, 2122-2132.

Martin, J.H., Friel, K.M., Salimi, I. \& Chakrabarty, S. (2007) Activity- and use-dependent plasticity of the developing corticospinal system. Neurosci Biobehav Rev, 31, 1125-1135. 
Milh, M., Kaminska, A., Huon, C., Lapillonne, A., Ben-Ari, Y. \& Khazipov, R. (2007) Rapid cortical oscillations and early motor activity in premature human neonate. Cereb Cortex, $17,1582-1594$.

Mima, T. \& Hallett, M. (1999a) Corticomuscular coherence: a review. J Clin Neurophysiol, 16, 501-511.

Mima, T. \& Hallett, M. (1999b) Electroencephalographic analysis of cortico-muscular coherence: reference effect, volume conduction and generator mechanism. Clin Neurophysiol, 110, 1892-1899.

Mima, T., Simpkins, N., Oluwatimilehin, T. \& Hallett, M. (1999) Force level modulates human cortical oscillatory activities. Neurosci Lett, 275, 77-80.

Müller, K., Ebner, B. \& Hömberg, V. (1994) Maturation of fastest afferent and efferent central and peripheral pathways: no evidence for a constancy of central conduction delays. Neurosci Lett, 166, 9-12.

Olivier, E., Edgley, S.A., Armand, J. \& Lemon, R.N. (1997) An electrophysiological study of the postnatal development of the corticospinal system in the macaque monkey. J Neurosci, $17,267-276$.

Omlor, W., Patino, L., Hepp-Reymond, M.C. \& Kristeva, R. (2007) Gamma-range corticomuscular coherence during dynamic force output. Neuroimage, 34, 1191-1198.

Petersen, T.H., Kliim-Due, M., Farmer, S.F. \& Nielsen, J.B. (2010) Childhood development of common drive to a human leg muscle during ankle dorsiflexion and gait. J Physiol, 588, 4387-4400.

Pitcher, J.B., Riley, A.M., Doeltgen, S.H., Kurylowicz, L., Rothwell, J.C., McAllister, S.M., Smith, A.E., Clow, A., Kennaway, D.J. \& Ridding, M.C. (2012a) Physiological evidence consistent with reduced neuroplasticity in human adolescents born preterm. J Neurosci, 32, 16410-16416.

Pitcher, J.B., Schneider, L.A., Burns, N.R., Drysdale, J.L., Higgins, R.D., Ridding, M.C., Nettelbeck, T.J., Haslam, R.R. \& Robinson, J.S. (2012b) Reduced corticomotor excitability and motor skills development in children born preterm. J Physiol, 590, 5827-5844.

Santiago-Rodríguez, E., León-Castillo, C., Harmony, T., Fernández-Bouzas, A. \& García-Gomar, M.L. (2009) Motor potentials by magnetic stimulation in periventricular leukomalacia. Pediatr Neurol, 40, 282-288.

Seth, A.K. (2008) Causal networks in simulated neural systems. Cogn Neurodyn, 2, 49-64.

Seth, A.K. (2010) A MATLAB toolbox for Granger causal connectivity analysis. J Neurosci Methods, 186, 262-273.

ten Donkelaar, H.J., Lammens, M., Wesseling, P., Hori, A., Keyser, A. \& Rotteveel, J. (2004) Development and malformations of the human pyramidal tract. J Neurol, 251, 1429-1442. 
Thorsteinsson, H., Reynisson, H.M., Sigurethardóttir, L.Y., Dagbjartsson, A. \& Karlsson, K.A. (2010) Behavioral correlates of direct current-coupled electrographic activity in premature infants. Behav Brain Res, 211, 11-15.

Tolonen, M., Palva, J.M., Andersson, S. \& Vanhatalo, S. (2007) Development of the spontaneous activity transients and ongoing cortical activity in human preterm babies. Neuroscience, 145, 997-1006.

Ushiyama, J., Suzuki, T., Masakado, Y., Hase, K., Kimura, A., Liu, M. \& Ushiba, J. (2011) Between-subject variance in the magnitude of corticomuscular coherence during tonic isometric contraction of the tibialis anterior muscle in healthy young adults. $\mathbf{J}$ Neurophysiol, 106, 1379-1388.

van der Meer, A.L., van der Weel, F.R. \& Lee, D.N. (1995) The functional significance of arm movements in neonates. Science, 267, 693-695.

von Hofsten, C. (2004) An action perspective on motor development. Trends Cogn Sci, 8, 266-272.

Witham, C.L., Riddle, C.N., Baker, M.R. \& Baker, S.N. (2011) Contributions of descending and ascending pathways to corticomuscular coherence in humans. J Physiol, 589, 3789-3800. 


\section{Figures and legends}

Figure 1

Hjorth derivation enhancing spatial resolution is preferable for computing the corticomuscular coherence. This method used mean voltage of four additional electrodes around the target area as reference, which is the primary sensorimotor area for the leg muscles $(\mathrm{Cz}$ according to the 10/20 international system).

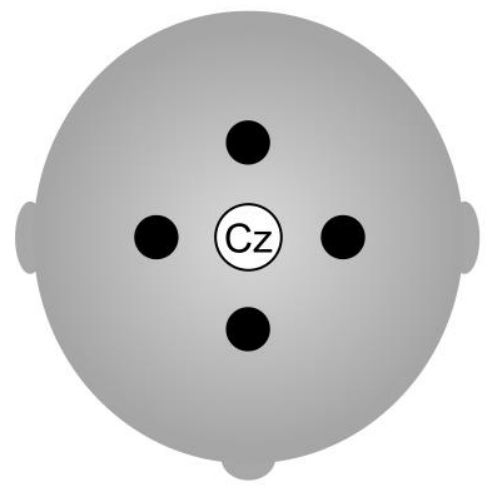

Figure 2

Recording setup. Experimenter held a neonate without any stimulation and restriction, so that the neonate moved his/her limbs spontaneously and freely during recording.

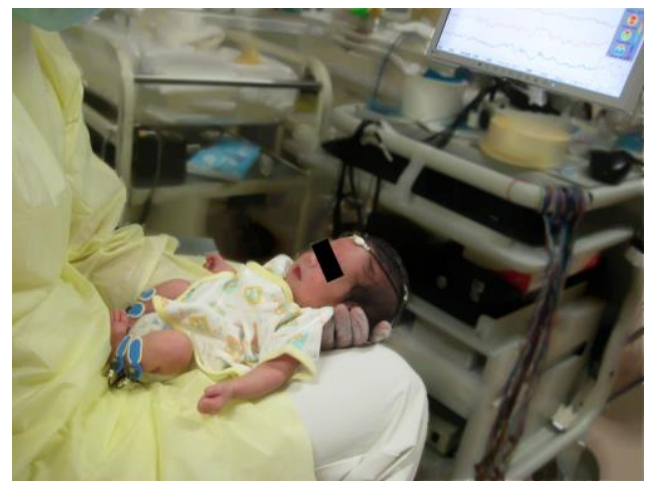

Figure 3

Representative EEG (upper) and EMG (lower) signals. Dotted lines indicate a period of TA muscle contraction. After excluding the initial and final $0.5 \mathrm{~s}$ transition periods, the rectangle (3 epochs) was selected for analysis in this graph.

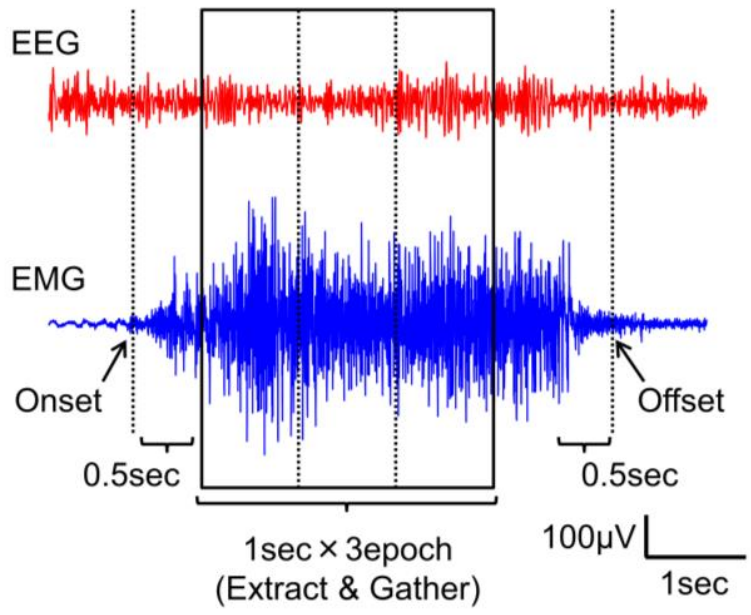


Figure 4

Representative data of EEG and EMG spectral powers

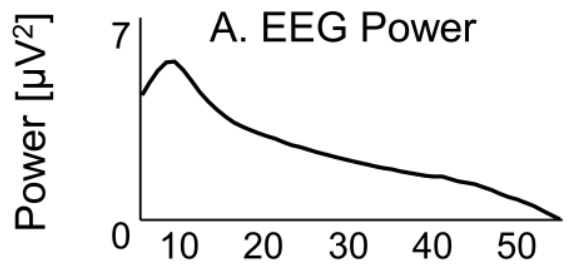

(A and $\mathrm{B}$ ), and the $\mathrm{CMC}(\mathrm{C})$. The dotted line indicates 95\% confidence limit. Thus, this subject has significant CMC between 25 and $30 \mathrm{~Hz}$.
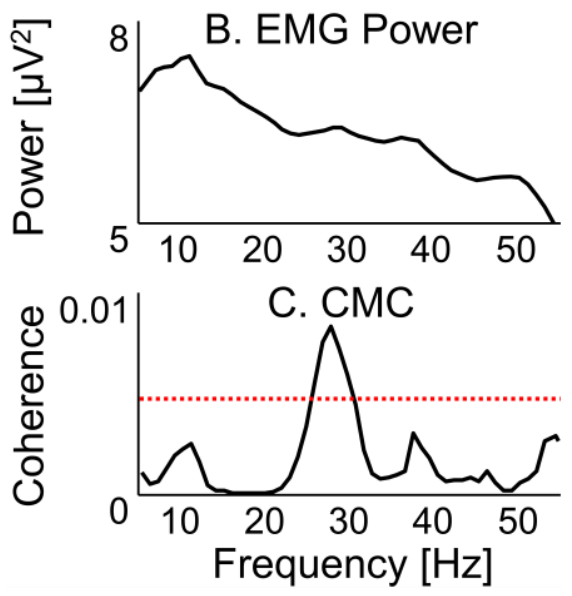

Figure 5

Relationship between CMC and postnatal

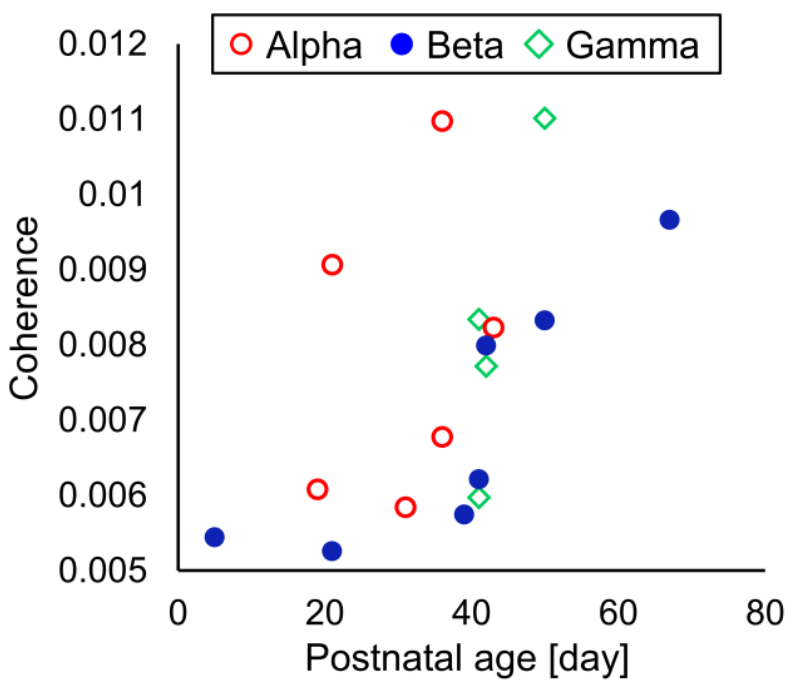

$0.67, \mathrm{p}=0.0055)$. 
Figure 6

Representative data of CMC and

Granger causality. Dotted lines in the each graph indicate the 95\% significance limits. Descending Granger causality is from EEG to EMG (middle graph), and ascending Granger causality is from EMG to EEG (lower graph). Thus, this neonate had unidirectional descending Granger causality.
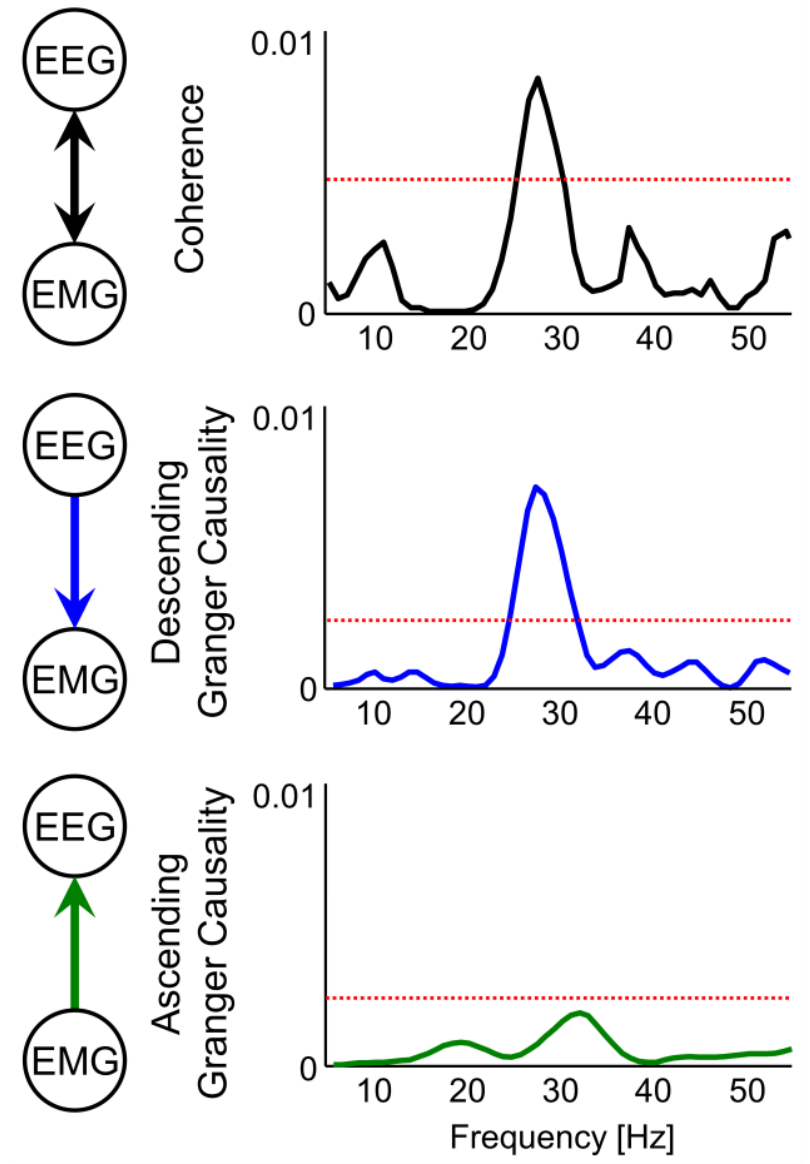
The published version is available on Wiley Online Library.

http://onlinelibrary.wiley.com/doi/10.1111/ejn.12612/abstract

Wiley Terms and Conditions for self-archiving

http://olabout.wiley.com/WileyCDA/Section/id-817011.html 